\title{
Cytomegalovirus pneumonia complicating immune checkpoint inhibitors-induced pneumonitis: A case report
}

\author{
OMAR BADRAN ${ }^{1}$, ANTON OURYVAEV $^{1}$, VERONIKA BATUROV $^{2}$ and AYELET SHAI ${ }^{1,3}$ \\ Departments of ${ }^{1}$ Oncology and ${ }^{2}$ Radiology, Galilee Medical Centre, Nahariya 22100; \\ ${ }^{3}$ Azriely Faculty of Medicine, Bar Ilan University, Zafed 1211502, Israel
}

Received October 15, 2020; Accepted February 11, 2021

DOI: $10.3892 / \mathrm{mco} .2021 .2282$

\begin{abstract}
A 63-year-old man was hospitalized for immune check-point inhibitors (ICIs) medicated pneumonitis, secondary to treatment with pembrolizumab for non-small cell lung cancer. He was treated with high dose steroids, mycophenolate mofetil, empiric broad spectrum antibiotics and empiric trimethoprim-sulfamethoxazole and intravenous immunoglobulin. Despite the aforementioned treatment, his condition continued to deteriorate. The patient was admitted to the intensive care unit. While intubated, he underwent bronchoscopy and lavage, which was analyzed for potential infectious agents. Cytomegalovirus (CMV) pneumonia was diagnosed and treated. He passed away despite antiviral treatment and maximal supportive care. CMV infection should be suspected in patients failing to recover from toxicities of ICIs with appropriate immunosuppression.
\end{abstract}

\section{Introduction}

Immune checkpoint inhibitors (ICIs), either alone or in combination, increase survival of patients with several types of malignant tumor and are currently being studied in the context of neoadjuvant and adjuvant care for multiple diseases. However, these monoclonal antibodies, targeting programmed cell death (PD)-1, PD-1 ligand (PDL-1) and cytotoxic T-lymphocyte antigen (CTLA)-4, are associated with a unique spectrum of adverse consequences that can affect virtually every organ in the body. These include autoimmune inflammation in the digestive tract, lung, skin, endocrine glands and peripheral and central nervous systems $(1,2)$. ICIs have been shown to increase survival of patients with metastatic non-small cell lung cancer (NSCLC) without activating genetic alteration in EGFR, ALK or reactive oxygen species and are now standard of care for these patients $(3,4)$.

Correspondence to: Dr Omar Badran, Department of Oncology, Galilee Medical Centre, 89 Meona Road, Nahariya 22100, Israel

E-mail: omarb@gmc.gov.il

Key words: non-small cell lung cancer, cytomegalovirus pneumonia, immunosuppressive therapy, immunotherapy
ICI-related pneumonitis (ICI-P) affects 3-5\% of patients treated with checkpoint inhibitors $(5,6)$. It appears to be more prevalent in patients with NSCLC than in those with other types of cancer $(6,7)$. Similarly, it is more common in patients treated with PD-1/PDL-1 inhibitors than in those treated with CTLA-4 inhibitors alone (6). The frequency of ICI-P is higher when anti-PD-1 and anti-CTLA-4 are administered concomitantly $(6,7)$. Despite the low fatality rate of $\sim 1 \%$, pneumonitis is one of the leading causes of ICI-associated morbidity (8).

Treatment of ICI-P depends on its severity: The majority of patients experience only mild to moderate pneumonitis and improve with withdrawal of immunotherapy and/or a course of corticosteroids $(9,10)$. However, certain patients worsen during treatment of pneumonitis and require additional immunosuppressive therapy, such as infliximab, mycophenolate mofetil $(9,10)$ or immunomodulatory agents, such as intravenous immunoglobulin (IVIG) (11). The clinical course may be further complicated by opportunistic infection, secondary to prolonged treatment with steroids and immunosuppressants $(12,13)$.

The clinical course of cytomegalovirus (CMV) pneumonia is typically indolent but fulminant disease may be observed in immunocompromised patients and carries a mortality risk mortality $>30 \%$ (14). CMV pneumonia has been infrequently described in patients receiving cancer immunotherapy. Here, we describe a patient that developed fatal CMV pulmonary infection following treatment for pneumonitis induced by the anti PD-1 antibody pembrolizumab.

\section{Case report}

A 63-year-old man with a medical history of chronic obstructive pulmonary disease, hyperlipidemia and diabetes mellitus was diagnosed with metastatic squamous cell lung carcinoma in May 2017 (Fig. 1). Tumor cells stained positively for PDL-1 with a count $>50 \%$, and the patient started treatment with pembrolizumab in June 2017.

In February 2019, a PET-CT scan showed no pathological uptake and the patient was in complete remission. The only side effect noted at that time was hypothyroidism, which was treated with levothyroxine.

In June 2019, the patient was admitted to Galilee Medical Center (Nahariya, Israel) due to respiratory distress and pulmonary infiltrates. He was treated with prednisone 


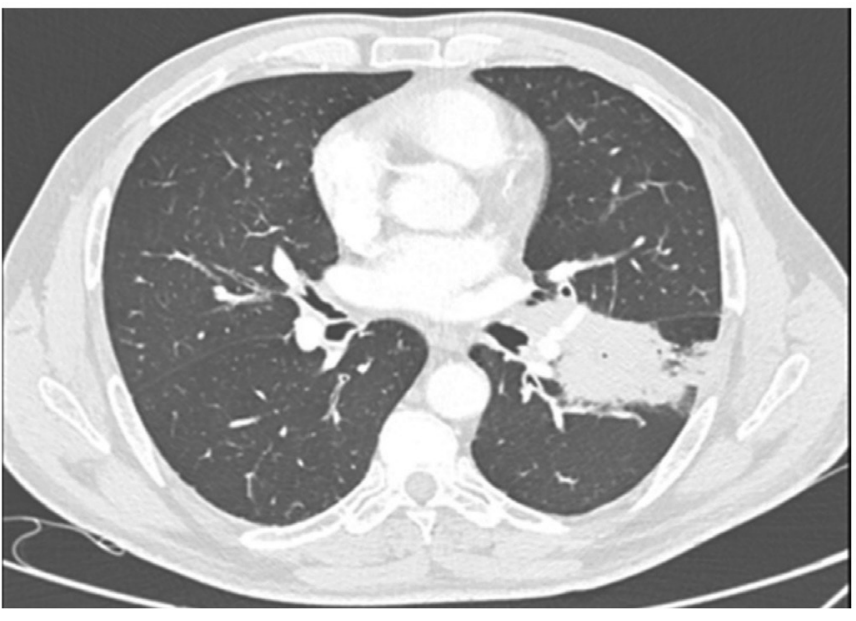

Figure 1. CT scan at diagnosis, showing left lung tumor mass.

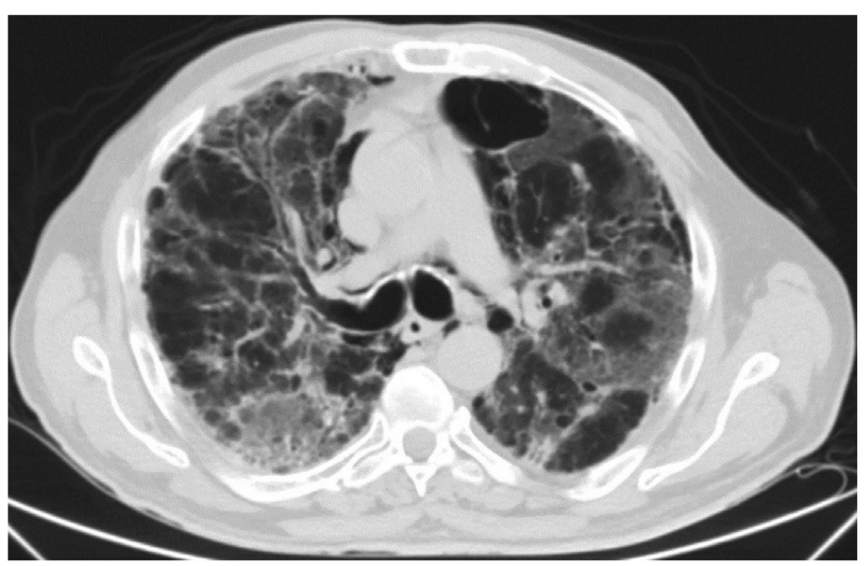

Figure 2. CT scan showing bilateral reticular opacity and disappearance of the left lung tumor mass.

( $1 \mathrm{mg} / \mathrm{kg}$ ) and levofloxacin and discharged following improvement with a recommendation to taper the dose of prednisone. While receiving $10 \mathrm{mg}$ prednisone, he experienced worsening dyspnea and was re-admitted.

On admission, the patient was tachypneic, but other vital signs were within the normal range. Blood oxygen saturation was $82 \%$ on ambient air. CT angiography demonstrated bilateral reticular opacities (Fig. 2).

The clinical course and imaging studies were consistent with grade 4 autoimmune pneumonitis secondary to ICIs in a patient with chronic obstructive pulmonary disease. The patient was treated with Prednisone 2 at a dose of $2 \mathrm{mg} / \mathrm{kg}$, mycophenolate mofetil, empiric broad-spectrum antibiotics and trimethoprim-sulfamethoxazole. Despite these measures, his condition worsened and the patient was intubated and mechanically ventilated due to respiratory failure.

The patient was admitted to the intensive care unit and IVIG was added to his treatment regimen. While intubated, he underwent bronchoscopy and lavage, which was analyzed for potential infectious agents. Following PCR analysis, CMV DNA was detected in the lavage fluid and CMV IgM was detected in the serum. Both tests supported the diagnosis of CMV pneumonia. Additionally, workup for Pneumocystis carinii (PCP), Ziehl-Neelsen staining and bacterial and fungal cultures were negative, and anti-viral treatment with Ganciclovir was added to his treatment regimen.

The patient was weaned off mechanical ventilation after two weeks and admitted to the Department of Oncology with high flow oxygen support and continued antiviral therapy, prednisone (slowly tapered to $0.5 \mathrm{mg} / \mathrm{kg}$ ) and prophylactic trimethoprim-sulfamethoxazole. However, despite these measures, his condition continued to deteriorate. After discussion with the patient and his family, the decision was made to avoid repeat intubation. The patient died 3 weeks later.

\section{Discussion}

We describe a patient that suffered from ICI-P complicated by CMV pneumonia, diagnosed by serology and a positive PCR test using pulmonary lavage. An autopsy with pathology review of lung tissue would constitute a definitive diagnostic test to support CMV pneumonia diagnosis, however the family declined this procedure. The clinical course, consisting of an initial improvement with steroids and subsequent deterioration and unresponsiveness to aggressive immune suppression, together with positive serological and PCR tests, support the diagnosis of CMV pneumonia in this patient. To the best of our knowledge, this is the first published report of CMV pneumonia complicating ICI-P.

Although the majority of patients with pneumonitis secondary to ICIs recover with corticosteroid treatment, fatalities may occur. It is important to recognize factors that may complicate the clinical course in these patients so they can be diagnosed and promptly treated. The clinical and radiological findings suggestive of CMV pneumonia are non-specific, overlapping with those of immune-mediated pneumonitis (15-17). Thus, a high degree of clinical suspicion is needed for early diagnosis. The present patient was treated with steroids for several weeks before diagnosis of CMV pneumonia, and doses were tapered, as aforementioned, suggesting that this complication can occur even after a relatively short course of steroids.

The range of clinical diseases due to CMV in immunocompromised patients is broad and includes hepatitis, retinitis, encephalitis, esophagitis and colitis (18). Our case demonstrates that workup for CMV infection should be administered in patients failing to improve with steroids for immune-mediated toxicity.

We report a case of fatal CMV pneumonia complicating ICI-P. A high degree of clinical suspicion and prompt evaluation for CMV infection is recommended in cases that do not improve with steroids for treatment of immunotherapy-associated adverse effects and in patients that relapse following initial improvement.

\section{Acknowledgements}

Not applicable.

\section{Funding}

No funding was received. 


\section{Availability of data and materials}

Not applicable.

\section{Authors' contributions}

AS was responsible for conception of the work, data acquisition and analysis, revising and finalizing the manuscript. OB was responsible for data acquisition and analysis, drafting and revising the manuscript. $\mathrm{AO}$ was responsible for acquisition and analysis.

\section{Ethics approval and consent to participate}

Not applicable.

\section{Patient consent for publication}

Not applicable.

\section{Competing interests}

The authors declare that they have no competing interests.

\section{References}

1. De Velasco G, Je Y, Bossé D, Awad MM, Ott PA, Moreira RB, Schutz F, Bellmunt V, Sonpavde GP, Hodi FS and Choueiri TK: Comprehensive meta-analysis of key immune-related adverse events from CTLA-4 and PD-1/PD-L1 inhibitors in cancer patients. Cancer Immunol Res 5: 312-318, 2017.

2. Postow MA, Sidlow R and Hellmann MD: Immune-related adverse events associated with immune checkpoint blockade. N Engl J Med 378, 158-168, 2018.

3. Gandhi L, Rodríguez-Abreu D, Gadgeel S, Esteban E, Felip E, De Angelis F, Domine M, Clingan P, Hochmair MJ Powell SF, et al: Pembrolizumab plus chemotherapy in metastatic non-small-cell lung cancer. N Engl J Med 378, 2078-2092, 2018.

4. Yuan M, Huang LL, Chen JH, Wu J and Xu Q: The emerging treatment landscape of targeted therapy in non-small-cell lung cancer. Signal Transduct Target Ther 4: 61, 2019.

5. Wang H, Guo X, Zhou J, Li Y, Duan L, Si X, Zhang L, Liu X, Wang M, Shi J and Zhang L: Clinical diagnosis and treatment of immune checkpoint inhibitor-associated pneumonitis. Thorac Cancer 11: 191-197, 2020.
6. Cadranel J, Canellas A, Matton L, Darrason M, Parrot A, Jean-Marc N, Lavolé A, Anne-Marie R and Fallet V: Pulmonary complication for immune checkpoint inhibitors in patient with nonsmall cell lung cancer. Eur Respir Revc 28: 190058, 2019.

7. Ma K, Lu Y, Jiang S, Tang J, Li X and Zhang Y: The relative risk and incidence of immune checkpoint inhibitors related pneumonitis in patients with advanced cancer: A meta-analysis. Front Pharmacol 9: 1430, 2018.

8. Topalian SL, Hodi FS, Brahmer JR, Gettinger SN, Smith DC, McDermott DF, Powderly JD, Carvajal RD, Sosman JA, Atkins MB, et al: Safety, activity, and immune correlates of anti-pd-1 antibody in cancer. N Engl J Med 366: 2443-2454, 2012.

9. Naidoo J, Page DB, Li BT, Connell LC, Schindler K, Lacouture ME, Postow MA and Wolchok JD: Toxicities of the anti-PD-1 and anti-PD-L1 immune checkpoint antibodies. Annals of Oncology. Ann Oncol 26: 2375-2391, 2015.

10. Brahmer JR, Lacchetti C, Schneider BJ, Atkins MB, Brassil KJ, Caterino JM, Chau I, Ernstoff MS, Gardner JM, Thompson JA, et al: Management of immune-related adverse events in patients treated with immune checkpoint inhibitor therapy: American society of clinical oncology clinical practice guideline. J Clin Oncol 36: 1714-1768, 2018.

11. Petri CR, Patell R, Batalini F, Rangachari D and Hallowell RW: Severe pulmonary toxicity from immune checkpoint inhibitor treated successfully with intravenous immunoglobulin: Case report and review of the literature. Respir Med Case Rep 27: 100834, 2019

12. Stuck AE, Minder CE and Frey FJ: Risk of infectious complications in patients taking glucocorticosteroids. Rev Infect Dis 11: 954-963, 1989

13. Fishman JA: Opportunistic infections-coming to the limits of immunosuppression. Cold Spring Harb Perspect Med 3: a015669, 2013.

14. Konoplev S, Champlin RE, Giralt S, Ueno NT, Khouri I, Raad I and Whimbey E: Cytomegalovirus pneumonia in adult autologous blood and marrow transplant recipients. Bone Marrow Transplantationc 27: 877-881, 2001.

15. Travi G and Pergam SA: Cytomegalovirus pneumonia in hematopoietic stem cell recipients. J Intensive Care Med 29: 200-212, 2014.

16. Franquet T, Lee KS and Müller NL: Thin-section CT findings in 32 immunocompromised patients with Cytomegalovirus pneumonia who do not have AIDS. Am J Roentgenol 181: 1059-1063, 2003.

17. Kang EY, Patz EF Jr and Müller NL: Cytomegalovirus pneumonia in transplant patients: CT findings. J Comput Assist Tomogr 20: 295-299, 1996.

18. Ljungman P, Boeckh M, Hirsch HH, Josephson F, Lundgren J, Nichols G, Pikis A, Razonable RR, Miller V and Griffiths PD: Definitions of cytomegalovirus infection and disease in transplant patients for use in clinical trials. Clin Infect Dis 64: 87-91, 2017. 